\title{
Cationic Polythiophene-based Colorimetric Assay for Probing the Activity of Protein Kinase A
}

\author{
Shanshan LIU, ${ }^{\dagger}$ Sitian ZHENG, and Jing CHU \\ Hubei Key Laboratory of Processing and Application of Catalytic Materials, College of Chemistry and Chemical \\ Engineering, Huanggang Normal University, Huanggang 438000, China
}

\begin{abstract}
In this work, a novel colorimetric assay based on polythiophene derivative (PMNT) was designed for the detection of protein kinase A (PKA). PKA can catalyze the phosphorylation of peptide, leading to the conformation change of PMNT from random-coil to planar, with the disappearance of absorption peaks above $500 \mathrm{~nm}$ and a color change from pink to yellow. The fabricated assay exhibits a wide linear range of $0.05-20 \mathrm{U} / \mathrm{mL}$ with a detection limit of $0.02 \mathrm{U} / \mathrm{mL}$ for PKA activity detection. The proposed protocol has promising prospects for use in clinical diagnosis related to PKA activity.
\end{abstract}

Keywords Conjugated polymers, protein kinase A, colorimetric, ATP

(Received October 31, 2020; Accepted November 21, 2020; Advance Publication Released Online by J-STAGE November 27, 2020)

\section{Introduction}

Protein kinase A (PKA), which can catalyze the phosphorylation process of proteins or peptide substrate containing tyrosine, threonine or serine, plays a crucial role in physiological processes involved in signal transduction, gene expression and cellular regulation. ${ }^{1-3}$ Its abnormal activity is widely observed in diseases including cancer, diabetes, cardiovascular diseases, and Alzheimer's disease. ${ }^{4-6}$ It is hence of great significance to develop simple, convenient, low-cost and sensitive methods for accurate detection of PKA activity.

There are methods that are based on radiometric, ${ }^{7}$ colorimetric,${ }^{8}$ electrochemical, ${ }^{9-12}$ photoelectrochemical, ${ }^{13,14}$ and fluorometric ${ }^{15,16}$ techniques for the monitoring of PKA activity. Although these assays have their own superiority, they suffer from drawbacks. For example, the radiometric approaches must address the hazardous effect of radioactive waste, whereas the electrochemical and photoelectrochemical methods are accompanied by tedious electrode modification and inferior stability, which may affect detection accuracy. Other techniques are mostly labor intensive and/or require sophisticated equipment. The colorimetric approach, with the advantages of simple operation, convenience, lowcost and practicality, has attracted much attention in the field of biosensors, and various colorimetric assays have been developed for PKA activity detection. For example, Song et al. designed platinum/carbon dot hybrid nanoparticles as catalyst to oxidize 3,3',5,5'tetramethylbenzidine (TMB) for ALP detection. ${ }^{8}$ Nie et al. reported a gold nanoparticle colorimetric assay with the aid of exopeptidase for sensitive detection of PKA activity. ${ }^{17}$ However, these methods have shortcomings, such as the introduction of additional chromogenic agents, unstable noble metal nanocluster or complex interactions of multiple enzymes. Therefore, there is still an urgent need to develop an easy-to-operate and cost-

$\dagger$ To whom correspondence should be addressed.

E-mail: liushanshanlhl@163.com effective colorimetric method for sensitive detection of PKA activity.

Conjugated polymers with robust light-harvesting ability and optical signal amplification effect ${ }^{18,19}$ have been utilized in bioimaging ${ }^{20-22}$ and biosensing. ${ }^{23,24}$ Water-soluble polythiophene derivatives display irregular curly conformation and exbibit a yellow color in their natural state. Once a relative planar conformation formed, polythiophene aqueous solution will turn pink in color. Based on the color change of polythiophene, Wang et al. constructed a strategy for the visual detection of DNA cleavage by means of the interaction between polythiophene and single-stranded DNA. ${ }^{25}$ Shinkai et al. developed a sensitive colorimetric assay based on the conformation change of polythiophene derivative for the detection of adenosine triphosphate (ATP). ${ }^{26}$ Since the phosphorylation of peptide substrate is accompanied by the transformation of ATP into ADP, it is envisaged that a simple biosensor for the detection of PKA activity can be developed based on the conformational change of polythiophene mediated by ATP.

In this work, we fabricated a novel colorimetric assay for PKA activity relying on the interaction between polythiophene and ATP. (Scheme 1). Cationic poly [3-( $3^{\prime}-N, N, N$-trimethylamino-1'-propyloxy)-4-methyl-2,5 thiophene hydrochloride] $(\mathrm{PMNT})^{25}$ was chosen as the optical probe, which was synthesized by oxidative polymerization according to the procedure reported in earlier literature. ${ }^{27}$ Once PMNT is mixed with ATP, the negatively charged phosphate group in ATP will bind with the positively charged ammonium group in PMNT through electrostatic interaction, and the PMNT/ATP complex will be produced. In the absorption spectrum of PMNT, new absorption peaks at 540 and $580 \mathrm{~nm}$ appear, and the solution color changes from yellow to pink. After the addition of PKA into the mixture of peptide substrate and PMNT/ATP complex, phosphorylation of the peptide substrate by PKA converts ATP to ADP. ADP contains less phosphate group and negative charges, which discourages further binding with PMNT, leading to the recovery of PMNT from planar conformation. Meanwhile, 


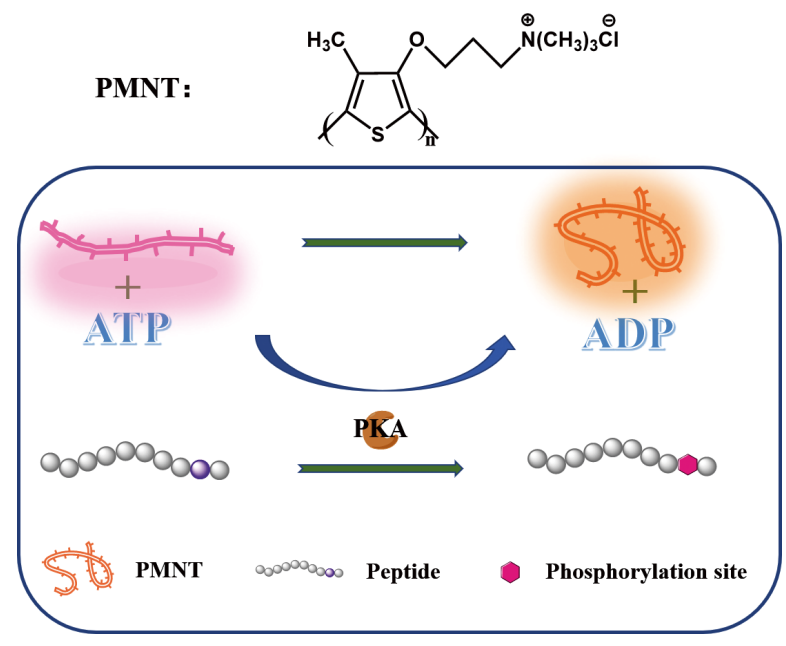

Scheme 1 Schematic of label-free colorimetric assay for protein kinase A detection method based on cationic polythiophene.

the absorption peaks at 540 and $580 \mathrm{~nm}$ disappeared, and the solution color recovered from pink to yellow. Taking advantage of conformational changes, a label-free, simple, cost-effective and sensitive assay for the test of PKA activity has been developed. Compared with the reported literature, ${ }^{28,29}$ this protocol eliminates the tedious operation of labeling organic small molecules on substrate peptide, the need to perform additional chromogenic reactions, amplified instability and biological reagents interference. The biosensor exhibits satisfactory performance in the detection of PKA activity, showing promising prospects for use in PKA-related clinical diagnosis and inhibitor screening.

\section{Experimental}

\section{Reagents and chemicals}

The substrate peptide (-LRRASLG-) was purchased from Sangon Biological Engineering Technology Co., Ltd (Shanghai). Poly[3-(3'-N,N,N-trimethylamino-1'-propyloxy)-4-methyl-2,5 thiophene hydrochloride] (PMNT) was synthesized according to the reported literature. ${ }^{27}$ Protein Kinase A (PKA) and bovine serum albumin (BSA) were from Sigma (USA). Alkaline phosphatase (ALP) and thrombin were purchased from Aladdin (Shanghai). Sodium hydroxide $(\mathrm{NaOH})$, magnesium chloride $\left(\mathrm{MgCl}_{2}\right)$, potassium chloride $(\mathrm{KCl})$, acetic acid and Tris(hydroxymethyl)aminomethane (Tris) were purchased from Sinopharm Chemical Reagent Co., Ltd. (Shanghai, China). All the reagents were of analytical grade and directly used without further purification. Ultrapure water obtained from a water purification system $(18.25 \mathrm{M} \Omega \mathrm{cm}$, UPT-II-10T, Ulupure Corporation, China) was used in all experiments.

\section{Apparatus}

Ultraviolet-visible (UV-vis) absorption spectra were recorded using a UV 2600 spectrophotometer (Shimadzu, Japan). The pH measurement of Tris-HCl buffer solution was taken by FE28 pH meter (Mettler Toledo, Shanghai, China).

\section{Protein kinase A activity assay}

First, $1.2 \mathrm{mg}$ of PMNT powder was dissolved in $10 \mathrm{~mL}$ of Tris buffer solution ( $2 \mathrm{mM}$ Tris, $\mathrm{pH} 7.2$, containing $0.5 \mathrm{mM}$ $\mathrm{MgCl}_{2}$ ). Then, $1.0 \mathrm{mg}$ of substrate peptide was dissolved in
$1.3 \mathrm{~mL} \mathrm{5 \%}$ aqueous acetic acid solution to obtain $1 \mathrm{mM}$ peptide stock solution for further use. Meanwhile, PKA solutions of designated concentrations were prepared. Then, $10 \mu \mathrm{L}$ of the prepared PMNT solution and $10 \mu \mathrm{L} 1.2 \mathrm{mM}$ ATP were added to $80 \mu \mathrm{L}$ of Tris buffer solution at $37^{\circ} \mathrm{C}$. After $1 \mathrm{~min}, 10 \mu \mathrm{L}$ of PKA solution of a particular concentration and $10 \mu \mathrm{L}$ of substrate peptide stock solution were dissolved into the above mixture and then made up to a volume of $300 \mu \mathrm{L}$, and the mixture was incubated at $37^{\circ} \mathrm{C}$ for $30 \mathrm{~min}$. Afterward, the obtained suspension was immediately transferred to a disposable cuvette for the collection of ultraviolet absorption spectra in the $300-650 \mathrm{~nm}$ range.

\section{Inhibition assay}

The effects of H-89 (a typical PKA inhibitor) on the activity of PKA was investigated. PKA $(10 \mathrm{nM})$, substrate peptide $(30 \mu \mathrm{M})$, ATP $(40 \mu \mathrm{M})$ and $\mathrm{H}-89$ with different final concentrations $(0.1,1,10,100,200,500,1000,2000 \mathrm{nM})$ were incubated at $37^{\circ} \mathrm{C}$ for $30 \mathrm{~min}$. Then, $10 \mu \mathrm{L}$ of the prepared PMNT solution was added and measurements were carried out according to the procedure previously described.

\section{Specificity assay}

In order to investigate the selectivity of the proposed assay, we used other interfering proteins such as BSA, p300, ALP and thrombin as contrast. To a mixture of PMNT, ATP and substrate peptide was added $10 \mu \mathrm{L}$ of enzyme (BSA, ALP, p300, thrombin) at $37^{\circ} \mathrm{C}$ and the resultant mixture was kept at $37{ }^{\circ} \mathrm{C}$ for $30 \mathrm{~min}$ before the collection of ultraviolet absorption spectra in the $300-650 \mathrm{~nm}$ range.

\section{Results and Discussion}

PMNT was synthesized following the procedure described in previous reported literature. ${ }^{27}$ The ${ }^{1} \mathrm{H}$ NMR spectrum is shown in Fig. S1 in Supporting Information. The Mn and PDI values of PMNT were 15391 and 1.33 , respectively. To verify the feasibility of PMNT to discriminate ADP from ATP, the optical responses of PMNT to ATP and ADP were recorded. As shown in Fig. 1A, the PMNT in Tris buffer solution displayed maximum absorption at $400 \mathrm{~nm}$ (Fig. 1A curve a). After the addition of ATP, new absorption peaks of PMNT appeared at 540 and $580 \mathrm{~nm}$ with a distinct color change from yellow to pink (Fig. 1A curve b; Fig. 1B a and b). The obvious change in absorption peak and color change are characteristic of the aggregation of polythiophene backbone ( $\pi$-stacked aggregates).$^{25}$ That is, the ATP could combine with PMNT to form PMNT/ ATP complex through electrostatic and hydrophobic interaction, forcing PMNT to transform from random-coil conformation to relative planar conformation. ${ }^{26}$ In order to explore the effect of concentration effect on the conformational change of PMNT, we changed the concentration of PMNT and collected the absorption spectrum of PMNT/ATP complex. As shown in Fig. S2, absorption peaks at 540 and $580 \mathrm{~nm}$ can appear at both high and low concentrations, indicating that ATP can induce conformational changes of PMNT, independent of the concentration of PMNT. In the presence of ADP, the absorption spectrum stays almost unchanged with only the PMNT solution, indicating that ADP alone has little effect on the absorption of PMNT (Fig. 1A, curve c; Fig. 1B, c). Moreover, we calculated the binding constants of PMNT for ATP and ADP from absorbance changes of PMNT by ATP and ADP, which were about $9.87 \times 10^{-2}$ and $4.1 \times 10^{-3} \mu \mathrm{M}$, respectively. Meanwhile, other ions have a negligible effect on the absorbance spectrum 

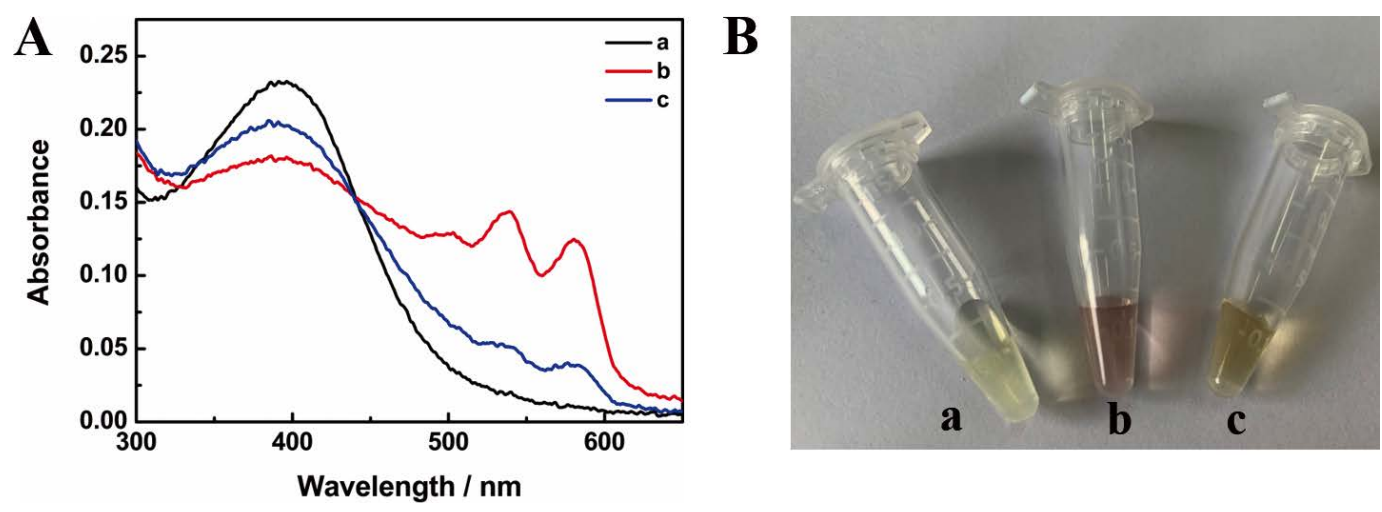

Fig. 1 (A) Absorption spectra and (B) photographs of PMNT before (a) and after incubating with ATP (b) and ADP (c). All measurements were taken in Tris buffer solution ( $2 \mathrm{mM}$ Tris, $0.5 \mathrm{mM} \mathrm{MgCl}_{2}$, $\mathrm{pH}$ 7.2).
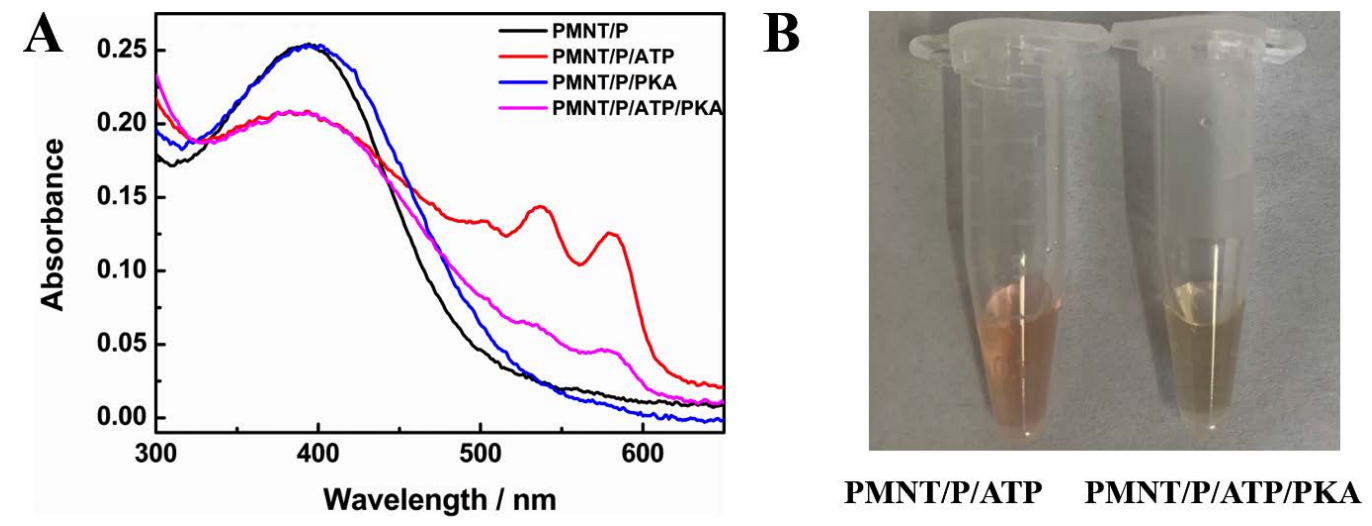

Fig. 2 (A) Absorption spectra of PMNT/Peptide, PMNT/Peptide/ATP, PMNT/Peptide/PKA, PMNT/ Peptide/ATP/PKA in Tris buffer containing $0.5 \mathrm{mM} \mathrm{MgCl}_{2}$. (B) Photographs of PMNT/P/ATP before and after incubating with PKA.

of PMNT (Fig. S3). These phenomena confirm the feasibility of the optical probe PMNT for the response to ATP. The good optical response to ADP and ATP guarantees sensitive detection of PKA in the subsequent experiments of the developed assay in this study.

The feasibility of the proposed method for PKA detection was also assessed. As shown in Fig. 2A, in the presence of substrate peptide, there is almost no influence on the absorption spectrum of the PMNT solution (curve a). After the addition of ATP into the above mixture, the characteristic absorption peak of the aggregation of polythiophene backbone appeared at 540 and $580 \mathrm{~nm}$, indicating that substrate peptide did not affect the electrostatic and hydrophobic interaction between ATP and PMNT. The addition of PKA to PMNT/P/ATP causes a blue shift from absorbance above 500 to $400 \mathrm{~nm}$ and returns the color of the mixture from pink back to yellow (Fig. 2B). These changes indicate that PKA could successfully catalyze the phosphorylation of peptide substrate and convert ATP to ADP. In addition, the experimental data of the control group show that by itself PKA has only weak influence on the absorption of PMNT/P. These phenomena confirm the feasibility of the proposed assay system for the detection of PKA activity.

The concentration of ATP was a significant factor influencing the detection performance. As shown in Fig. 3A, the absorbance intensity of PMNT/ATP at $540 \mathrm{~nm}$ gradually increases with increasing amount of ATP from 0 to $40 \mu \mathrm{M}$ and almost leveled at $40 \mu \mathrm{M}$. The intensity at $100 \mu \mathrm{M}$ is $c a$. $105 \%$ of that at
$40 \mu \mathrm{M}$. Considering phosphorylation efficiency, $40 \mu \mathrm{M}$ was chosen as the optimal ATP concentration for the following experiments.

In order to obtain high catalytic efficiency, the phosphorylation reaction time was s to optimized. As depicted in Fig. 3B, the absorbance intensity at $540 \mathrm{~nm}$ dramatically decreases with time and plateaus after $30 \mathrm{~min}$. Therefore, $30 \mathrm{~min}$ was chosen as the best reaction time for the PKA-catalyzed reaction.

Under the optimal conditions, the developed colorimetric assay was tested for quantitative determination of PKA activity. Figure 4A shows the absorbance response of the PMNT after incubating with ATP and substrate peptide, having the PKA concentration varied from 0 to $100 \mathrm{U} / \mathrm{mL}$. Obviously, with the addition of PKA, the absorbance intensity at 540 and $580 \mathrm{~nm}$ decreased as a consequence of planar conformation formation, which is linearly proportional to the activity of PKA within the PKA concentration range of $0.05-20 \mathrm{U} / \mathrm{mL}$. The calibration curve was constructed by plotting $\Delta$ Abs. $(\Delta$ Abs. $=$ Abs. $.0-$ Abs.; where Abs.0 is the absorbance response when the PKA concentration is zero and Abs. is the current response when the PKA were at different concentrations) versus different PKA concentrations. The fitted linear regression equation is Abs. $=0.058 \log C+0.038$ with a correlation coefficient of 0.991 (Fig. 4B). The detection limit was estimated to be $0.02 \mathrm{U} / \mathrm{mL}$ at $3 \sigma$ criterion. The linear range was wider and the detection limit is comparable with those of previous works (Table 1). ${ }^{11,15,28-32}$ This indicates that the fabricated method exhibits good analytical 

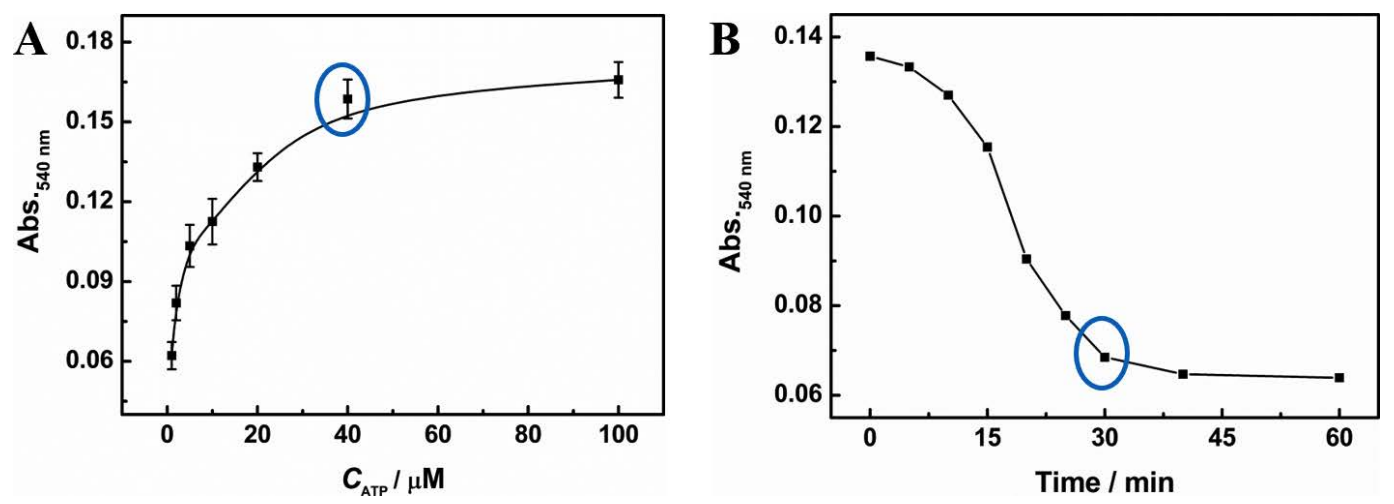

Fig. 3 (A) Absorption intensity of PMNT with ATP concentrations varied from 0 to $100 \mu \mathrm{M}$. (B) Absorbance intensity of PMNT/ATP versus time in PKA catalyzed reaction $\left(C_{\mathrm{PKA}}=80 \mathrm{U} / \mathrm{mL}\right)$.
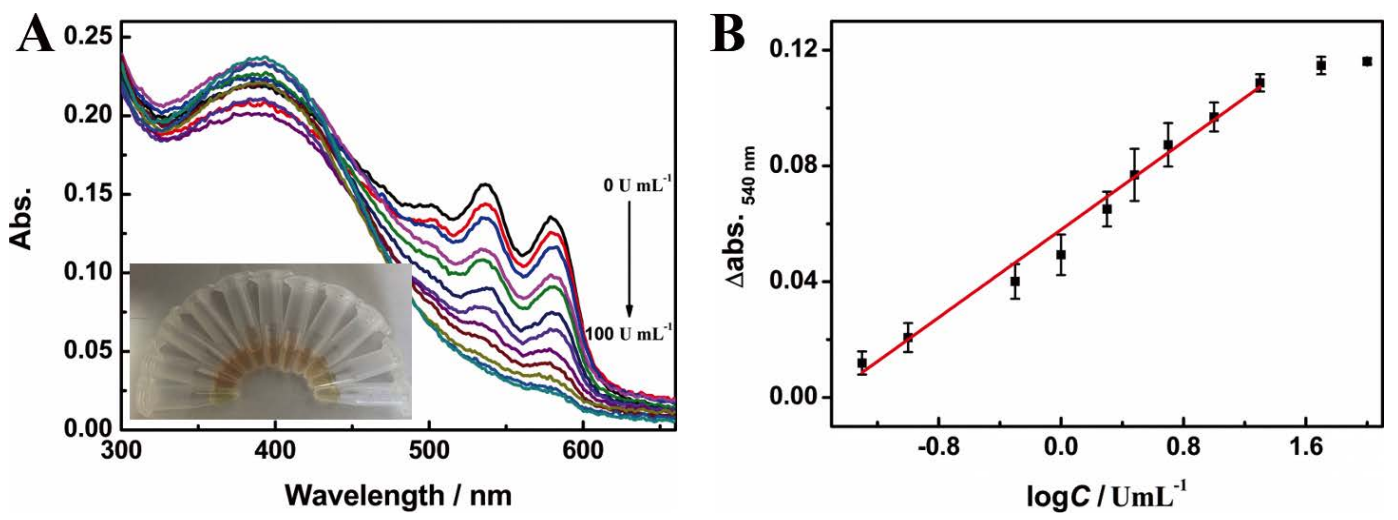

Fig. 4 (A) Absorbance response of the derived biosensor to PKA with concentration varied from 0 to $100 \mathrm{U} / \mathrm{mL}$. The inset shows the changes in the color of the PMNT/P/ATP/PKA mixture. (B) Linear calibration curve of absorption changes versus PKA concentration.

Table 1 Performance comparison of the proposed assay with previously reported systems for the detection of PKA

\begin{tabular}{lclc}
\hline \multicolumn{1}{c}{ Method } & $\begin{array}{c}\text { Linear range/ } \\
\mathrm{U} \mathrm{mL}^{-1}\end{array}$ & $\begin{array}{c}\text { Detection } \\
\text { limit }\end{array}$ & Reference \\
\hline Colorimetry & $0-1.0$ & $0.232 \mathrm{U} \mathrm{mL}^{-1}$ & 15 \\
Colorimetry & - & $10 \mathrm{nM}$ & 28 \\
Colorimetry & - & $14 \mathrm{nM}$ & 29 \\
Electrochemistry & $0.1-10$ & $0.09 \mathrm{U} \mathrm{mL}^{-1}$ & 30 \\
Photoelectrochemical & $0.05-100$ & $0.17 \mathrm{U} \mathrm{mL}^{-1}$ & 11 \\
Fluorescence & $1-2000$ & $0.47 \mathrm{U} \mathrm{mL}^{-1}$ & 31 \\
ECL & $0.05-100$ & $0.17 \mathrm{U} \mathrm{mL}^{-1}$ & 32 \\
This work & $0.05-20$ & $0.02 \mathrm{U} \mathrm{mL}^{-1}$ & This work \\
\hline
\end{tabular}

performance for PKA activity assay.

As protein kinase inhibitors could reduce the aberrant expression and activity of PKA, it is of great significance to assess the feasibility of the developed assay in inhibitor screening. H-89, a typical PKA inhibitor, was selected as the model. In the presence of H-89 (Fig. S5), there is a gradual increase of absorbance intensity at $540 \mathrm{~nm}$ with an increase in the concentration of $\mathrm{H}-89$, indicating that $\mathrm{H}-89$ could influence the activity of $\mathrm{H}-89$. The plot of $\Delta \mathrm{Abs}$. versus $\mathrm{H}-89$ concentration is depicted in Fig. 5. The calculated IC50 (half maximal inhibitory concentration) is $c a .37 \mathrm{nM}$, which was consistent with previous reports, ${ }^{15,33}$ suggesting that the developed assay based on the PMNT/ATP probe has the ability of screening

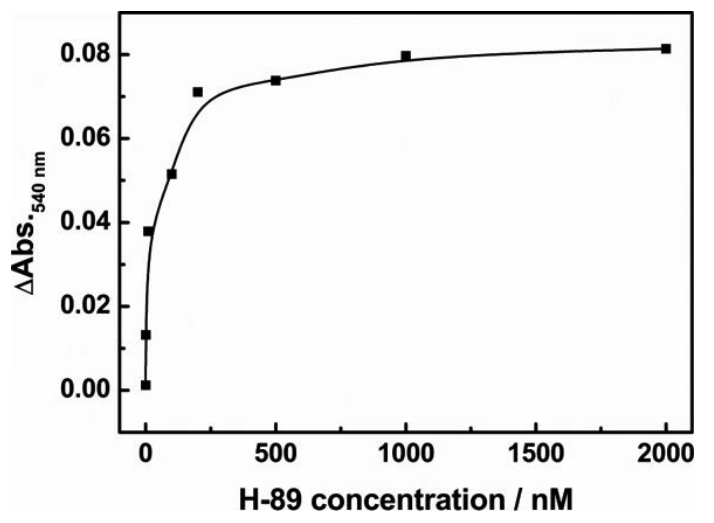

Fig. 5 Inhibition effect of $\mathrm{H}-89$ on PKA activity, from bottom to top: $0.1,1,10,100,200,500,1000$ and $2000 \mathrm{nM}$.

PKA inhibitors.

To evaluate the specificity of the developed assay, the absorbance intensity of interferential proteins, including ALP, BSA, p300 and thrombin, were collected under the optimized conditions. As shown in Fig. 6, only PKA can dramatically reduce the absorbance and other proteins have almost no influence on the absorbance of PMNT/P/ATP, indicating the excellent selectivity of the proposed method for PKA activity detection. In other words, the interferential proteins failed to catalyze the phosphorylation reaction of substrate peptide. 


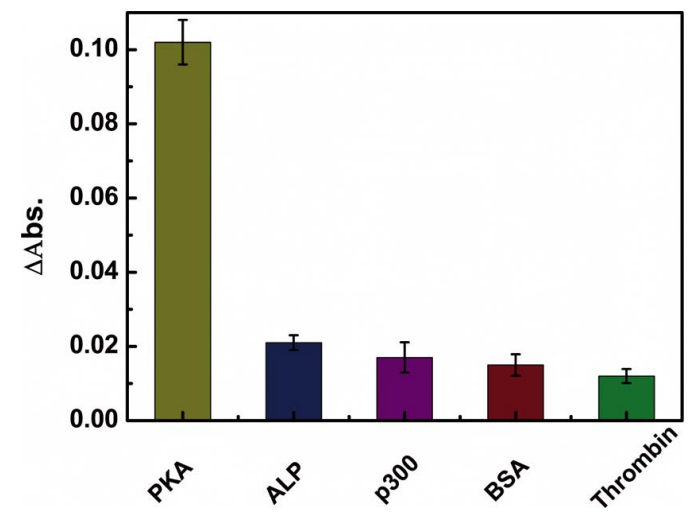

Fig. 6 Specificity of the developed assay among different proteins. (PKA: $80 \mathrm{U} / \mathrm{mL}$, other proteins: $2.0 \mu \mathrm{M}$ )

Furthermore, parallel measurements carried out for six independent samples gave a relative standard deviation of $8.3 \%$, which suggests good reproducibility and satisfactory analytical performance.

\section{Conclusions}

In summary, we have constructed a novel colorimetric biosensor for sensitive detection of PKA activity based on the conformational change of cationic polythiophene. The absorbance of PMNT with positive charge ammonium groups led to the appearance of new absorption peaks in the presence of ATP through electrostatic interaction and hydrophobic effect, but ADP had almost no effect on that of PMNT. Taking advantage of the response difference among PMNT, ATP and ADP, PKA activity can be monitored with high sensitivity. Comparing with other colorimetric methods, the proposed protocol is simple, rapid and convenient with no need of additional chromogenic reaction and tedious labeling procedures. With a broad linear range, the assay exhibits excellent selectivity and superior sensitivity, covering PKA concentration range of $0.05-20 \mathrm{U} / \mathrm{mL}$, and showing a detection limit as low as $0.02 \mathrm{U} / \mathrm{mL}$. As such, the developed detection platform has great potential for use in PKA-related clinical diagnosis.

\section{Acknowledgements}

This work was supported by the the National Natural Science Foundation of China (22004044), Doctoral Fund Project of Huanggang Normal University (2042019027) and Research Foundation of Huanggang Normal University (204201913103).

\section{Supporting Information}

This material is available free of charge on the Web at http:// www.jsac.or.jp/analsci/.

\section{References}

1. T. Hunter, Cell, 2000, 100, 113.

2. B. E. Kemp and R. B. Pearson, Trends Biochem. Sci., 1990, $15,342$.

3. Y. Nishizuka, Nature, 1984, 308, 693.
4. H. Cho, J. Mu, J. K. Kim, J. L. Thorvaldsen, Q. Chu, E. B. Crenshaw, K. H. Kaestner, M. S. Bartolomei, G. I. Shulman, and M. J. Birnbaum, Science, 2001, 292, 1728.

5. M. Flajolet, G. He, M. Heiman, A. Lin, A. C. Nairn, and P. Greengard, Proc. Natl. Acad. Sci. U. S. A., 2007, 104, 4159.

6. J. S. Sebolt-Leopold and R. Herrera, Nat. Rev. Cancer, 2004, 4, 937.

7. C. J. Hastie, H. J. Mclauchlan, and P. Cohen, Nat. Protoc., 2006, 1,968 .

8. Y. Z. Wang, W. J. Qi, and Y. J. Song, Chem. Commun., 2016, 52, 7994.

9. Q. Hu, J. M. Kong, D. X. Han, Y. Bao, X. J. Zhang, Y. W. Zhang, and L. Niu, Talanta, 2020, 206, 120173.

10. Q. X. Luo, Y. Li, R.-P. Liang, S.-P. Cao, H.-J. Jin, and J.-D. Qiu, J. Electroanal. Chem., 2020, 856, 113706.

11. Q. Hu, Q. Wang, C. Jiang, J. Zhang, J. Kong, and X. Zhang, Biosens. Bioelectron., 2018, 110, 52.

12. L. P. Jia, R. N. Zhao, L. J. Wang, R. N. Ma, W. Zhang, L. Shang, and H. S. Wang, Biosens. Bioelectron., 2018, 117, 690

13. H. S. Yin, B. Sun, L. F. Dong, B. C. Li, Y. L. Zhou, and S. Y. Ai, Biosens. Bioelectron., 2015, 64, 462.

14. Y. Wang, X. Li, G. I. N. Waterhouse, Y. L. Zhou, H. S. Yin, and S. Y. Ai, Talanta, 2019, 196, 197.

15. C. C. Jia, J. Bai, Z. P. Liu, S. T. Gao, Y. M. Han, and H. Y. Yan, Anal. Chim. Acta, 2020, 1128, 99.

16. S. H. Hewitt, J. Parris, R. Mailhot, and S. J. Butler, Chem. Commun., 2017, 53, 12626

17. J. Zhou, X. H. Xu, X. Liu, H. Li, Z. Nie, M. Qing, Y. Huang, and S. Z. Yao, Biosens. Bioelectron., 2014, 53, 295

18. C. L. Zhu, L. B. Liu, Q. Yang, F. T. Lv, and S. Wang, Chem. Rev., 2012, 112, 4687.

19. W. Huang, E. Smarsly, J. S. Han, M. Bender, K. Seehafer, I. Wacker, R. R. Schroder, and U. H. F. Bunz, ACS Appl. Mater. Interfaces, 2017, 9, 3068.

20. L. H. Liu, Q. Zhang, J. Wang, L.L. Zhao, L. X. Liu, and Y. Lu, Talanta, 2019, 198, 128.

21. K. Narasimha and M. Jayakannan, Macromolecules, 2016, $49,4102$.

22. H. Wang, L. Y. Zhou, C. C. Zhou, W. W. Zhao, J. W. Wang, L. B. Liu, S. Wang, and Y. L. Wang, ACS Appl. Mater. Interfaces, 2017, 9, 23544.

23. Z.Yuan, Y. Y. Zhou, S. X. Gao, Y. Q. Cheng, and Z. P. Li, ACS Appl. Mater. Interfaces, 2014, 6, 6181.

24. F. T. Araujo, L. O. Peres, and L. Caseli, Langmuir, 2019 , 35, 7294.

25. Y. L. Tang, F. D. Feng, F. He, S. Wang, Y. L. Li, and D. B. Zhu, J. Am. Chem. Soc., 2006, 128, 14972.

26. C. Li, M. Numata, M. Takeuchi, and S. Shinkai, Angew. Chem. Int. Ed., 2005, 44, 6371.

27. H. A. Ho, M. Boissinot, M. G. Bergeron, G. Corbeil, K. Dore, D. Boudreau, and M. Leclerc, Angew. Chem. Int. Ed., 2002, 41, 1548.

28. H. Sotound, P. Gribbon, B. Ellinger, J. Reinshagen, P. Boknik, L. Kattner, A. Ei-Armouche, and T. Eschenhagen, J. Biomol. Screen., 2013, 18, 899.

29. K.-Y. Tomizaki and H. Mihara, Mol. Biosyst., 2006, 2, 580.

30. Z. Wang, Z. Yan, N. Sun, and Y. Liu, Biosens. Bioelectron., 2015, 68, 771 .

31. C. Shen, X. Xia, S. Hu, M. Yang, and J. Wang, Anal. Chem., 2015, 87, 693.

32. Q. X. Luo, Y. Li, R. P. Liang, S. P. Cao, H. J. Jin, and J. D. Qiu, J. Electroanal. Chem., 2020, 856, 113706.

33. Q. Hu, J. M. Kong, D. X. Han, Y. Bao, X. J. Zhang, Y. W. Zhang, and L. Niu, Talanta, 2020, 206, 120173. 